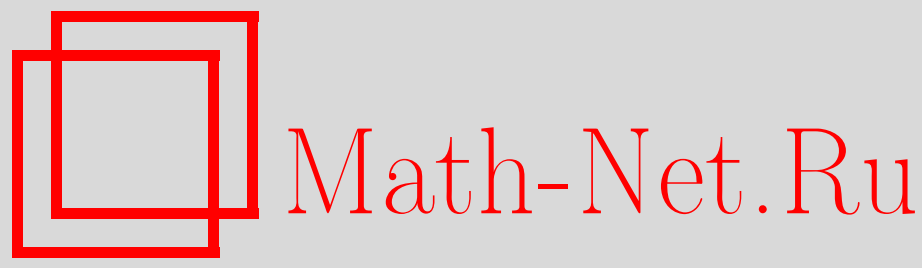

А. А. Туганбаев, Автоморфизм-продолжаемые модули, Дискрет. матем., 2015, том 27, выпуск 2, 106-111

DOI: https://doi.org/10.4213/dm1328

Использование Общероссийского математического портала Math-Net.Ru подразумевает, что вы прочитали и согласны с пользовательским соглашением http://www . mathnet.ru/rus/agreement

Параметры загрузки:

IP: 54.81 .137 .203

26 апреля 2023 г., 14:36:52

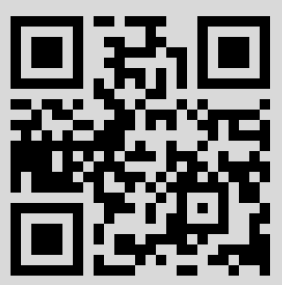




\title{
Автоморфизм-продолжаемые модули
}

\author{
() 2015 г. А. А. Туганбаев*
}

Исследуются модули, у которых все автоморфизмы подмодулей продолжаются до эндоморфизмов (автоморфизмов) всего модуля.

Автор поддержан Российским фондом фундаментальных исследований. Проект 08-01-00693-а: Структурная теория колец, проект 14-01-000452-А.

Ключевые слова: автоморфизм-продолжаемый модуль, автоморфизм-инвариантный модуль, сильно автоморфизм-продолжаемый модуль.

Данная работа является продолжением статьи [9]. Все кольца предполагаются ассоциативными и с ненулевой единицей, а модули унитарными. Подмодуль $M$ модуля $E$ называется характеристическим, если $\alpha(M) \subseteq M$ для каждого автоморфизма $\alpha$ модуля $E$.

Рассмотрим три условия на модуль $M$.

I) $M$ - автоморфизм-инвариантный модуль, т.е. $M$ - характеристический подмодуль своей инъективной оболочки. Автоморфизм-инвариантные модули изучались в ряде работ; см., например, [1], [2], [3], [5], [6], [10], [12]. В [2; Theorem 16] доказано, что модуль $M$ является автоморфизм-инвариантным в точности тогда, когда $M-$ псевдоинбективный модуль, т.е. если для любого подмодуля $X$ в $M$ каждый мономорфизм $X \rightarrow M$ продолжается до эндоморфизма модуля $M$. Псевдоинъективные модули изучались в ряде работ; см., например, [4], [7], [2].

II) $M$ - сильно автоморфизм-продолжаемый модуль, т.е. для любого подмодуля $X$ в $M$ каждый автоморфизм модуля $X$ продолжается до автоморфизма модуля $M$.

III) $M$ - автоморфизм-продолжаемый модуль, т.е. для любого подмодуля $X$ в $M$ каждый автоморфизм модуля $X$ продолжается до эндоморфизма модуля $M$. Автоморфизм-продолжаемые и сильно автоморфизм-продолжаемые модули изучались в работах [9] и [11].

Замечание 1. Импликация I) $\Rightarrow$ II) доказана в лемме 3 данной работы, а импликация II) $\Rightarrow$ III) очевидна. В общем случае импликация II) $\Rightarrow$ I) не верна. Действительно, аддитивная группа рациональных чисел $\mathbb{Q}$ является инъективной оболочкой модуля $\mathbb{Z}$ над кольцом целых чисел $\mathbb{Z}$. Кроме того, $\mathbb{Z}$ не является автоморфизм-инвариантным модулем, поскольку $\alpha(\mathbb{Z}) \not \mathbb{Z}$, где $\alpha: q \rightarrow q / 2$ - автоморфизм $\mathbb{Z}$-модуля $\mathbb{Q}$. Тем не менее, непосредственно проверяется, что любой нетождественный автоморфизм $\alpha$ произвольного ненулевого подмодуля $X$ модуля

*Место работы: Национальный исследовательский университет "МЭИ", e-mail: tuganbaev@gmail.com 
$\mathbb{Z}_{\mathbb{Z}}$ является умножением на -1 ; поэтому $\alpha$ продолжается до автоморфизма модуля $\mathbb{Z}_{\mathbb{Z}}$.

Замечание 2. Пусть $A$ - кольцо и $M$ - правый $A$-модуль. Автору не известно, верна ли импликация III) $\Rightarrow$ II) для произвольного модуля $M$. В [11] доказано, что импликация III) $\Rightarrow$ II) верна в следующих случаях:

1) $M=X \oplus T$, где $X$ - несингулярный модуль, $T$ - инъективный модуль (в частности, импликация III) $\Rightarrow$ II) верна для несингулярных модулей);

2) $A$ - ограниченное нетерово первичное кольцо. В частности, импликация III) $\Rightarrow$ II) верна для модулей над коммутативными дедекиндовыми кольцами.

Замечание 3. Если $M$ - артинов (например, конечный) модуль, то по теореме 2 из [9] $M$ является автоморфизм-продолжаемым модулем в точности тогда, когда $M$ - автоморфизм-инвариантный модуль.

В связи с замечаниями 1, 2 и 3 мы сформулируем теорему 1 , которая применима к конечным кольцам и является основным результатом данной работы.

Теорема 1. Пусть $A-$ кольцо.

1) Если $A$ полупримарно (например, если кольцо $A$ конечно), то автоморбизм-продолжаемые А-модули совпадают c автоморфизм-инвариантными модулями.

2) Если кольио А нетерово справа, то автоморфизм-продолжсаемые правые A-модули совпадают с сильно автоморфизм-продолжсаемыми модулями.

Доказательство теоремы 1 разбито на ряд утверждений, некоторые из которых представляют самостоятельный интерес. Приведем необходимые определения и обозначения.

Модуль $M$ называется нетеровым, если $M$ не содержит бесконечных строго возрастающих цепей подмодулей. Слова типа " $A$ - нетерово кольцо" означают, что $A_{A}$ и ${ }_{A} A$ - нетеровы модули. Подмодуль $X$ модуля $M$ называется существенным, если $X \cap Y \neq 0$ для любого ненулевого подмодуля $Y$ из $M$; в этом случае $M$ называется существенным расширением подмодуля $X$. Модуль $E$ называется ингективным, если для любого модуля $X$ и каждого подмодуля $Y$ в $X$ любой гомоморфизм $Y \rightarrow E$ продолжается до гомоморфизма $X \rightarrow E$. Если $E-$ инъективный модуль и $E-$ существенное расширение модуля $M$, то $E$ - называется ингективной оболочкой модуля $M$. Прямые суммы простых модулей называются полупростыми модулями. Для модуля $M$ через $\operatorname{Soc}(M)$ обозначается цоколь модуля $M$, т.е. $\operatorname{Soc}(M)-$ наибольший полупростой подмодуль модуля $M(\operatorname{Soc}(M)=0$, если $M$ не содержит полупростых подмодулей). Говорят, что модуль $M$ имеет конечную цокольную длину $n$, если $M$ содержит такую строго возрастающую конечную цепь (цокольный ряд) подмодулей $M_{0} \subset M_{1} \subset \ldots \subset M_{n-1} \subset M_{n}$, что $M_{0}=0, M_{n}=M, M_{i} / M_{i-1}$ - цоколь модуля $M / M_{i-1}$, причем $M / M_{i-1}$ - существенное расширение ненулевого полупростого модуля $M_{i} / M_{i-1}, i=1, \ldots, n$. Кольцо $A$ называется наследственным справа, если каждый его правый идеал изоморфен прямому слагаемому прямой суммы изоморфных копий модуля $A_{A}$. Правый модуль $M$ над кольцом $A$ называется несингулярным, если $M$ не имеет таких ненулевых элементов $m$, что правый идеал $\{a \in A \mid m a=0\}$ является существенным правым идеалом в $A$. Кольцо $A$ называется ограниченным справа, если каждый его существенный правый идеал содержит ненулевой идеал кольца $A$. Кольцо $A$ называется первичным, если произведение 
любых двух его ненулевых идеалов не равно нулю. Кольцо $A$ называется $n о л у$ примарным, если его радикал Джекобсона нильпотентен, а фактор-кольцо $A / J(A)$ является полупростым кольцом.

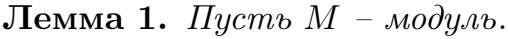

1) Для любого подмодуля $X^{\prime}$ в $M$ каждый эндоморфизм (соотв. автоморфизм) модуля $X^{\prime}$ продолжается до эндоморфизма (соотв. автоморфизма) некоторого существенного подмодуля $X$ в $M$.

2) $М$ является автоморфизм-продолжсаемым в точности тогда, когда для любого существенного подмодуля $X$ в $M$ каждый автоморфизм модуля $X$ продолжается до эндоморфизма модуля $M$.

3) $М$ является сильно автоморфизм-продолжаемым в точности тогда, когда для любого существенного подмодуля $X$ в $M$ каждый автоморбизм модуля $X$ продолжается до автоморфизма модуля $M$.

Доказательство. 1), 2) Утверждения доказаны в [9; Лемма 1].

3) Утверждение вытекает из 1).

Лемма 2. Пусть $M-$ модуль и $E$ - ингективная оболочка модуля $M$. Равносильны условия:

1) $M-$ автоморфизм-инвариантный модуль;

2) $M=\alpha(M)=\alpha^{-1}(M)$ для любого автоморфизма $\alpha$ модуля $E$;

3) каждый изоморфизм между любыми существенными подмодулями модуля $M$ продолжается до эндоморфизма модуля $M$;

4) каждый изоморфизм между любыми существенными подмодулями модуля $M$ продолжается до автоморфизма модуля $M$.

Доказательство. Импликации 1) $\Rightarrow 2$ ) и 4) $\Rightarrow$ 3) очевидны.

Импликация 2) $\Rightarrow 1$ ) следует из того, что $\alpha(M) \subseteq M$ и $\alpha^{-1}(M) \subseteq M$.

Эквивалентность 1) $\Leftrightarrow 3)$ доказана в [9; Лемма 2].

$2) \Rightarrow 4)$. Пусть $X$ и $Y$ - существенные подмодули модуля $M$ и $f: X \rightarrow Y$ - изоморфизм. Так как инъективная оболочка $E$ модуля $M$ является существенным расширением модуля $M$ и $M$ - существенное расширение модулей $X$ и $Y$, то $E-$ существенное расширение модулей $X$ и $Y$. Поскольку модуль $E$ инъективен, то $f$ продолжается до эндоморфизма $\alpha$ модуля $E$. Тогда $X \cap \operatorname{Ker}(\alpha)=\operatorname{Ker}(f)=0$. Поскольку $E$ - существенное расширение модуля $X$, то $\operatorname{Ker}(\alpha)=0, \alpha-$ мономорфизм и модуль $\alpha(E)$ инъективен. Тогда $\alpha(E)$ - прямое слагаемое модуля $E$ и $Y=f(X)=\alpha(X) \subseteq \alpha(E)$. Так как $E$ - существенное расширение модуля $Y$, то $\alpha(E)$ - существенное прямое слагаемое модуля $E$. Поэтому $E=\alpha(E)$ и $\alpha$ - автоморфизм модуля $E$. По условию 2) $M=\alpha(M)=\alpha^{-1}$. Поэтому $\alpha$ индуцирует автоморфизм $g$ модуля $M$, который является искомым продолжением изоморфизма $f$.

Лемма 3. Если $M$ - автоморфизм-инвариантный модуль, то $M-$ сильно автоморфизм-продолжаемый модуль.

Доказательство. Пусть $X$ - подмодуль в $M$ и $f$ - автоморфизм модуля $X$. Надо доказать, что $f$ продолжается до автоморфизма модуля $M$. По лемме $1(3)$ без ограничения общности можно считать, что $X$ - существенный подмодуль в $M$. Так как $f$ - изоморфизм существенного подмодуля $X$ автоморфизм-инвариантного модуля $M$ на существенный подмодуль $X$ в $M$, то по лемме $2 f$ продолжается до автоморфизма модуля $M$. 
Лемма 4. Пусть $M$ - автоморфизм-продолжаемый модуль и для любого эндоморфизма $h \in \operatorname{End}(M)$, ядро которого является существенным подмодулем в $M$, эндоморбизм $1_{M}-h$ модуля $M$ является автоморфизмом. Тогда $M-$ сильно автоморфизм-продолжаемый модуль.

Доказательство. Пусть $X$ - подмодуль в $M$ и $f$ - автоморфизм модуля $X$. Надо доказать, что $f$ продолжается до автоморфизма модуля $M$. По лемме $1(3)$ без ограничения общности можно считать, что $X$ - существенный подмодуль в $M$. Так как $M$ - автоморфизм-продолжаемый модуль, то $f$ и $f^{-1}$ продолжаются до эндоморфизмов $\alpha$ и $\beta$ модуля $M$ соответственно. Обозначим через $h_{1}$ и $h_{2}$ эндоморфизмы $1_{M}-\beta \alpha$ и $1_{M}-\alpha \beta$ модуля $M$ соответственно. Так как $h_{1}(X)=0=h_{2}(X)$, то $\operatorname{Ker}\left(h_{1}\right)$ и $\operatorname{Ker}\left(h_{2}\right)$ - существенные подмодули в $M$. Так как $\beta \alpha=1_{M}-h_{1}$ и $\alpha \beta=1_{M}-h_{2}$, то по условию $\beta \alpha$ и $\alpha \beta$ - автоморфизмы модуля $M$. Поэтому $\alpha$ - автоморфизм модуля $M$.

Лемма 5. Пусть $M$ - автоморфизм-продолжаемый модуль и для каждого элемента $x \in M$ и любого эндоморфизма $h \in \operatorname{End}(M)$, ядро которого является существенным подмодулем в $M$, существует такое натуральное число $n=n(x, h)$, что $h^{n}(x)=0$. Тогда $M-$ сильно автоморфизм-продолжаемый модуль.

Доказательство. Пусть $h \in \operatorname{End}(M)$ и $\operatorname{Ker}(h)$ - существенный подмодуль в $M$. По лемме 4 достаточно доказать, что эндоморфизм $1_{M}-h$ модуля $M$ является автоморфизмом. Составим формальный ряд $1_{M}+\sum_{k=1}^{\infty} h^{k}$. Так как для каждого элемента $x \in M$ существует такое натуральное число $n=n(x, h)$, что $h^{n}(x)=0$, то $1_{M}+\sum_{k=1}^{\infty} h^{k}-$ корректно определенный эндоморфизм модуля $M$. Непосредственно проверяется, что

$$
\left(1_{M}-h\right)\left(1_{M}+\sum_{k=1}^{\infty} h^{k}\right)=\left(1_{M}+\sum_{k=1}^{\infty} h^{k}\right)\left(1_{M}-h\right)=1_{M} .
$$

Поэтому $1_{M}-h$ - автоморфизм модуля $M$.

Предложение 1. Пусть $M$ - автоморфизм-продолжаемый модуль и каждый ицклический подмодуль модуля $M$ является нетеровым модулем. Тогда $M-$ сильно автоморфизм-продолжаемый модуль.

Доказательство. Пусть $X$ - произвольный ненулевой циклический подмодуль модуля $M$ и $h$ - такой эндоморфизм модуля $M$, что $\operatorname{Ker}(h)$ - существенный подмодуль в $M$. По лемме 5 достаточно доказать, что $h^{n}(X)=0$ для некоторого натурального числа $n$. Обозначим $X_{0}=0$ и $X_{i}=X \cap \operatorname{Ker}\left(h^{i}\right), i=1,2,3 \ldots$ Тогда $X_{i-1} \subseteq X_{i}$ и $h\left(X_{i}\right) \subseteq h\left(X_{i-1}\right), i=1,2,3 \ldots$ По условию $X$ - нетеров модуль. Поэтому существует такое натуральное число $n$, что $X_{n}=X_{n+1}$. Пусть $f: X \rightarrow M$ - ограничение гомоморфизма $h^{n}$ на модуль $X$. Так как $X_{n}=X \cap \operatorname{Ker}\left(h_{n}\right)=\operatorname{Ker}(f)$, то гомоморфизм $f$ индуцирует изоморфизм $g: X / X_{n} \rightarrow g\left(X / X_{n}\right) \subseteq M$. Так как $\operatorname{Ker}(h)$ - существенный подмодуль модуля $M$, то $\operatorname{Ker}(h) \cap g\left(X / X_{n}\right)$ - существенный подмодуль в $g\left(X / X_{n}\right)$. Поскольку $g: X / X_{n} \rightarrow g\left(X / X_{n}\right)$ - изоморфизм, то $g^{-1}\left(\operatorname{Ker}(h) \cap g\left(X / X_{n}\right)\right)$ - существенный подмодуль в $X / X_{n}$. Обозначим через $Y$ - полный прообраз в $X$ подмодуля $g^{-1}\left(\operatorname{Ker}(h) \cap g\left(X / X_{n}\right)\right)$ в $X / X_{n}$ при действии $g$. Тогда

$$
\begin{gathered}
h^{n+1}(Y)=h\left(h^{n}(Y)\right)=h(f(Y))=h\left(g\left(g^{-1}\left(\operatorname{Ker}(h) \cap g\left(X / X_{n}\right)\right)\right)\right) \subseteq \\
\subseteq h\left(\operatorname{Ker}(h) \cap g\left(X / X_{n}\right)\right)=0 .
\end{gathered}
$$


Поэтому $Y \subseteq X_{n+1}$ и $Y / X \subseteq X_{n+1} / X_{n}=0$. Тогда $\operatorname{Ker}(h) \cap g\left(X / X_{n}\right)=g(Y / X)=0$. Так как $\operatorname{Ker}(h) \cap g\left(X / X_{n}\right)$ - существенный подмодуль в $g\left(X / X_{n}\right)$, то $g\left(X / X_{n}\right)=0$. Поэтому $h^{n}(X)=f(X)=g\left(X / X_{n}\right)=0$, что и требовалось доказать.

Лемма 6. Если $M$ - сильно автоморфизм-продолжаемый модуль и $X$ - характеристический подмодуль в $M$, то $X$ - сильно автоморфизм-продолжаемый модуль.

Доказательство. Пусть $Y$ - подмодуль в $X$ и $f$ - автоморфизм модуля $X$. Так как $M$ - сильно автоморфизм-продолжаемый модуль, то $f$ продолжается до автоморфизма $\alpha$ модуля $M$. Поскольку $X$ - характеристический подмодуль в $M$, то автоморфизм $\alpha$ индуцирует требуемый автоморфизм $g$ модуля $X$, являющийся продолжением автоморфизма $f$ модуля $Y$.

Лемма 7. Пусть $M-$ модуль конечной цокольной длинъ $n$.

1) $h^{n}(M)=0$ для любого эндоморфизма $h \in \operatorname{End}(M)$ с существенным в $M$ ядром.

2) Если $M-$ автоморфизм-продолжаемый модуль, то $M-$ сильно автоморбизм-продолжаемый модуль.

Доказательство. 1) Будем использовать индукцию по $n$. При $n=1$ утверждение следует из того, что любой существенный подмодуль полупростого модуля совпадает со всем модулем. Допустим, что утверждение верно для модулей конечной цокольной длины $n-1$. Модуль $M$ конечной цокольной длины $n$ содержит такой подмодуль $X$, что $X$ - модуль конечной цокольной длины $n-1$ и $M / X$ - полупростой модуль. По предположению индукции $X \subseteq \operatorname{Ker}\left(h^{n-1}\right)$. Kроме того, $M / X$ - полупростой модуль. Поэтому $h^{n-1}(M)$ - полупростой модуль. Тогда $h^{n-1}(M)$ содержится в существенном подмодуле $\operatorname{Ker}(h)$ модуля $M$. Поэтому $h^{n}(M)=0$.

2) Утверждение следует из 1) и леммы 5.

Предложение 2. Если $M$ - автоморфизм-продолжаемый модуль конечной иокольной длины $n$, то $M$ - автоморфизм-инвариантный модуль.

Доказательство. Пусть $M \neq 0$. Будем вести индукцию по $n$. При $n=1 M-$ полупростой модуль; в частности, $M$ - автоморфизм-инвариантный модуль.

Допустим, что каждый автоморфизм-продолжаемый модуль конечной цокольной длины $n-1$ - автоморфизм-инвариантный модуль. Модуль $M$ содержит такую строго возрастающую конечную цепь подмодулей $M_{0} \subset M_{1} \subset \ldots \subset M_{n-1} \subset M_{n}$, что $M_{0}=0, M_{n}=M, M_{i} / M_{i-1}$ - цоколь модуля $M / M_{i-1}$, причем $M / M_{i-1}-$ существенное расширение полупростого модуля $M_{i} / M_{i-1}, i=1, \ldots, n$. Непосредственно проверяется, что $M_{n-1}$ - характеристический подмодуль в $M=M_{n}$. По лемме $7(2) M$ - сильно автоморфизм-продолжаемый модуль. По лемме $6 M_{n-1}-$ сильно автоморфизм-продолжаемый модуль конечной цокольной длины $n-1$. По предположению индукции $M_{n-1}$ - автоморфизм-инвариантный модуль. Пусть $E-$ инъективная оболочка модуля $M$ и $\alpha$-автоморфизм модуля $E$. Надо доказать, что $\alpha(M) \subseteq M$.

Так как $E$ - существенное расширение модуля $M$ и $M$ - существенное расширение модуля $M_{1}$, то $E$ - существенное расширение полупростого модуля $M_{1}$, лежащего в $M_{n-1}$. Поэтому $E$ - инъективная оболочка автоморфизм-инвариантного модуля $M_{n-1}$. Поэтому $M_{n-1}=\alpha\left(M_{n-1}\right)$ и $\alpha$ индуцирует автоморфизм $f$ подмодуля $M_{n-1}$ сильно автоморфизм-продолжаемого модуля $M$. Следовательно, $f$ продолжается до автоморфизма $g$ модуля $M$. Обозначим через $\beta$ - ограничение $\alpha$ на модуль $M$. Если $(\beta-g)(M)=0$, то $\alpha(M)=\beta(M)=g(M)=M$, что и требовалось. 
Допустим, что $(\beta-g)(M) \neq 0$. Обозначим $X=M_{1} \cap(\beta-g)(M) \subseteq M$. Так как $M_{1}$ - существенный подмодуль в $E$, то $X$ - существенный подмодуль ненулевого модуля $(\beta-g)(M)$. Кроме того, $(\beta-g)\left(M_{n-1}\right)=(f-g)\left(M_{n-1}\right)=0$. Пусть $Y-$ полный прообраз модуля $X$ в модуле $M$ при действии $\beta-g$. Так как $X-$ существенный подмодуль ненулевого модуля $(\beta-g)(M)$ и $(\beta-g)\left(M_{n-1}\right)=0$, то $Y / M_{n-1}$ - существенный подмодуль полупростого модуля $M / M_{n-1}$. Поэтому

$$
\begin{gathered}
M / M_{n-1}=Y / M_{n-1}, \quad M=Y, \\
\beta(M)=\beta(Y) \subseteq g(Y)+(\beta-g)(Y) \subseteq M+M_{1}=M .
\end{gathered}
$$

Тогда $\alpha(M)=\beta(M) \subseteq M$, что и требовалось.

Замечание 4. Хорошо известно, что каждый модуль над полупримарным кольцом является модулем конечной цокольной длины.

Окончание доказательства теоремы 1. Утверждение 1) теоремы 1 вытекает из замечания 4, предложения 2 и леммы 3. Утверждение 2) теоремы 1 вытекает из предложения 1 и того, что все циклические правые модули над нетеровыми справа кольцами являются нетеровыми модулями.

Замечание 5. Если $A$ - полупримарное кольцо, то автоморфизм-продолжаемые $A$-модули совпадают с сильно автоморфизм-продолжаемыми $A$-модулями.

Замечание 5 вытекает из леммы $7(2)$ и замечания 4.

\section{Список литературы}

1. Alahmadi A., Er N, Jain S.K., "Modules which are invariant under monomorphisms of their injective hulls", J. Australian Math. Soc., 79:3 (2005), 2265-2271.

2. Er N., Singh S., Srivastava A.K., "Rings and modules which are stable under automorphisms of their injective hulls", J. Algebra, 379 (2013), 223-229.

3. Guil Asensio P.A., Srivastava A.K., "Automorphism-invariant modules satisfy the exchange property", J. Algebra, 388 (2013), 101-106.

4. Jain S.K., Singh S., "Quasi-injective and pseudo-injective modules", Canadian Math. Bull., 18:3 (1975), 359-366.

5. Lee T.K., Zhou Y., "Modules which are invariant under automorphisms of their injective hulls", J. Algebra Appl., 6:2 (2013).

6. Singh S., Srivastava A.K., "Rings of invariant module type and automorphism-invariant modules", Contemp. Math., 609, 299-311.

7. Teply M.L., "Pseudo-injective modules which are not quasiinjective", Proc. Amer. Math. Soc., 49:2 (1975), 305-310.

8. Tuganbaev A., Semidistributive Modules and Rings, Kluwer Academic Publishers, Dordrecht-Boston-London, 1998.

9. Туганбаев А.А., "Автоморфизмы подмодулей и их продолжение”, Дискрет. матем., 25: 1 (2013), 144-151.

10. Туганбаев А.А., "Характеристические подмодули инъективных модулей”, Дискрет. матем., 25:2 (2013), 85-90.

11. Туганбаев А.А., "Продолжения автоморфизмов подмодулей", Фундаменталъная и прикладная математика, 18: 3 (2013), 179-198.

12. Туганбаев А.А., "Характеристические подмодули инъективных модулей над строго первичными кольцами", Дискрет. матем., 26: 3 (2014), 121-126. 